\title{
PHOTOLUMINESCENCE OF DONOR-DOPED ZnSe FILMS GROWN BY MOLECULAR BEAM EPITAXY
}

\author{
G. KARCZEWSKI \\ Institute of Physics, Polish Academy of Sciences \\ Al. Lotników 32/46, 02-668 Warszawa, Poland
}

\author{
B. Hu, A. Yin, H. Luo, M. Dobrowolska and J.K. Furgyna \\ Department of Physics, University of Notre Dame, Notre Dame, Indiana 46556, USA
}

\begin{abstract}
We studied the effect of the donor doping of ZnSe films on their photoluminescence properties. The samples were doped during the molecular beam epitaxy growth, either with gallium or with chlorine. As the dopant concentration dose increases, the intensity of the band-edge emission first saturates, and then quenches in favor of the deep-level photoluminescence band. The main effect of donor doping on photoluminescence is a strong increase in intensity of the donor-bound exciton line, referred to as $I_{2}$. For Ga-doped films deep-band emission is much stronger, and the $I_{2}$-line is slightly weaker than for Cl-doped films with comparable doping level. The results confirm the superiority of chlorine over gallium as an $n$-type dopant in $\mathrm{ZnSe}$. We discuss the photoluminescence results and relate them to deep level transient spectroscopy data obtained on the same samples.
\end{abstract}

PACS numbers: 68.55.Ln, 78.55.Et

Since most of the proposed applications of $\mathrm{ZnSe}$ are based on the concept of $p-n$ junction, the problem of the influence of doping on basic properties of this material has to be addressed systematically. In our earlier papers we reported on the deep level transient spectroscopy (DITS) investigations of deep states in $n$-type gallium- and chlorine-doped ZnSe epilayers grown by molecular beam epitaxy (MBE) $[1,2]$. In the current investigation we focus on photoluminescence properties of these materials.

Gallium is most frequently used as a donor dopant for $\mathrm{ZnSe}[3,4]$. It substitutes for $\mathrm{Zn}$ in the $\mathrm{ZnSe}$ lattice. Unfortunately, high $\mathrm{Ga}$ concentration in $\mathrm{ZnSe}$ results in saturation of the carrier concentration, at the level of about $10^{18} \mathrm{~cm}^{-3}[5]$. In addition, doping with $\mathrm{Ga}$ appears to be harmful to optoelectronic device performance, because it causes a significant decrease in the near-band-gap photoluminescence in favor of deep-level optical transitions. As an alternative to $\mathrm{Ga}$ atoms, group-VII elements can be employed for doping of $\mathrm{ZnSe} \mathrm{[6-8].} \mathrm{They} \mathrm{substitute}$ 
for Se in the ZnSe lattice. One of the group-VII elements - chlorine - has already been shown to be very effective as an $n$-type dopant. The highest carrier concentrations ever reached in $\mathrm{ZnSe}-$ exceeding $10^{19} \mathrm{~cm}^{-3}-$ have recently been reported for $\mathrm{ZnSe:Cl}$ grown by $\mathrm{MBE}[7]$.

In this investigation the doped $\mathrm{ZnSe}$ films were grown on GaAs (100) substrates. The source materials were $\mathrm{Zn}$ and $\mathrm{Se}$, both of $6 \mathrm{~N}$ purity. The substrate temperature was kept at $T_{\mathrm{s}}=300^{\circ} \mathrm{C}$. All $\mathrm{ZnSe}$ epilayers were of the same thickness, $d=2 \mu \mathrm{m}$. For the Cl-doping we employed $\mathrm{ZnCl}_{2}$ as the source, and for Ga-doping we used elemental gallium source. The net donor concentrations in the films were evaluated from the $C-V$ characteristics.

Photoluminescence (PL) measurements were performed at low temperatures $(T=10 \mathrm{~K})$. The PL spectra were excited by the $384 \mathrm{~nm}$ emission line of an Art-ion laser operated at low power densities $\left(0.9 \mathrm{~mW} / \mathrm{cm}^{2}\right)$, and measured using a SPEX monochromator with photon-counting electronics.

In Fig. 1 we compare the PL spectra obtained on an undoped $\mathrm{ZnSe}$ film with those observed on Cl-doped layers with different chlorine concentrations. A

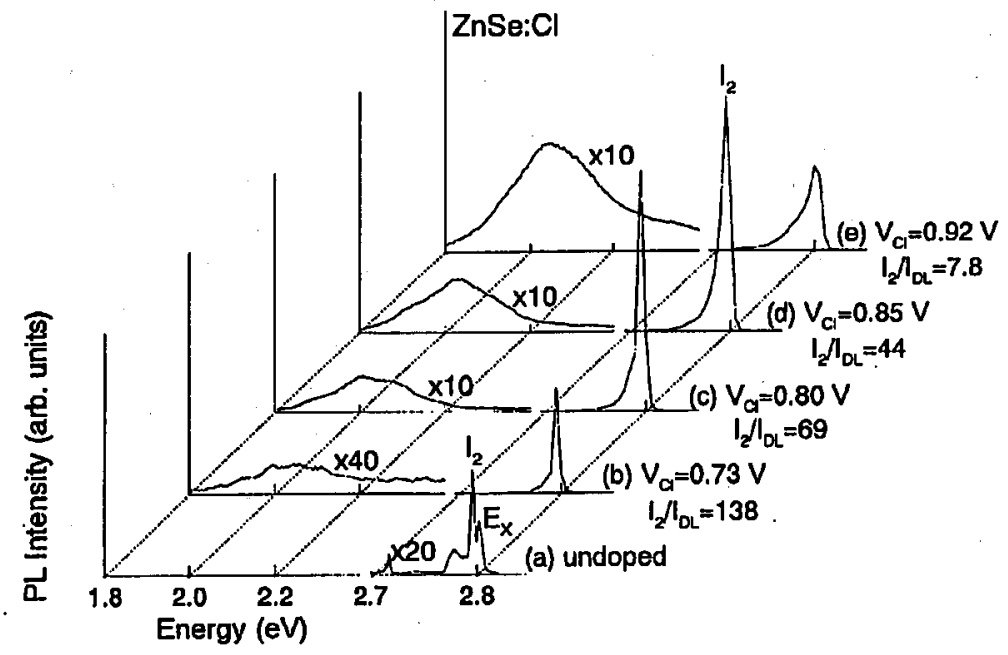

Fig. 1. Photoluminescence spectra of undoped (a) and chlorine-doped (b)-(e) $\mathrm{ZnSe}$.

typical luminescence spectrum of an undoped $\mathrm{ZnSe}$ epilayer (1a) is dominated by two distinct emission lines in the band-edge region - the neutral donor-bound exciton line (marked $I_{2}$ ) at $2.796 \mathrm{eV}$, and the free-exciton line (marked $E_{x}$ ) at $2.802 \mathrm{eV}$. A much weaker bound exciton line associated with a deep-acceptor $\left(I_{1}^{\text {deep }}\right)$ at $2.78 \mathrm{eV}$ signifies the presence of a number of deep acceptors, which partially compensate the native donors which dominate the electronic properties of undoped $\mathrm{ZnSe}$. Chlorine doping causes a strong increase in the bound exciton line $I_{2}$ and the disappearance of the free exciton emission $E_{x}$.

The increase in the intensity of the $I_{2}$-line observed for moderate doping levels indicates a significant increase in the shallow donor concentration due to 
Cl-doping. In the case of the sample with the lowest Cl-doping, the intensity of the $I_{2}$-line increases about 20 times with respect to the intensity of that line in the undoped film. For higher doping doses the intensity of the blue band-edge emission first saturates, and then starts to quench in favor of the deep photoluminescence band seen around $2.0 \mathrm{eV}$ - the so-called "orange emission". The intensity of this orange emission, $I_{\mathrm{DL}}$, increases steadily with the Cl-doping. This $2.0 \mathrm{eV}$ photoluminescence band is usually attributed to a self-activated center, possibly related to zinc vacancies $\mathrm{V}_{\mathrm{Zn}_{\mathbf{n}}}$ [9].

Figure 2 provides a comparison of the photoluminescence spectra obtained on $\mathrm{ZnSe}$ epilayers doped with $\mathrm{Ga}$ and with $\mathrm{Cl}$ of nearly the same net donor concentrations, $3 \times 10^{17} \mathrm{~cm}^{-3}$. There are two major differences between the PL spectra

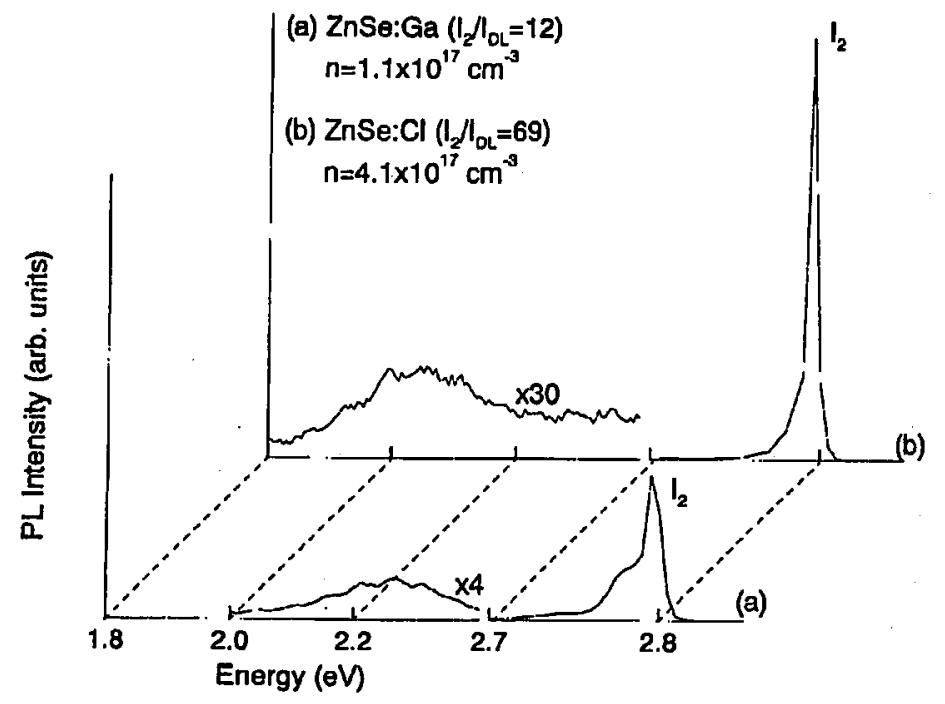

Fig. 2. Photoluminescence spectra (a) of gallium-doped and (b) chlorine-doped ZnSe. The doping level in both cases was approximately $3 \times 10^{17} \mathrm{~cm}^{-3}$.

shown in Fig. 2. First, for Ga-doped films the deep-band emission is much stronger, and the $I_{2}$-line is slightly weaker than for Cl-doped films. As a result, the ratio $I_{2} / I_{\mathrm{DL}}$ is about six times higher for $\mathrm{ZnSe}: \mathrm{Cl}$ than for $\mathrm{ZnSe}: \mathrm{Ga}$. Second, the deep level (DL) band in $\mathrm{ZnSe}: \mathrm{Ga}$ occurs at a higher energy (around 2.2-2.3 eV) than in the case of $\mathrm{ZnSe}: \mathrm{Cl}$ (around $2.0 \mathrm{eV}$ ).

The comparison of the PL results presented above with the recent DLTS data $[1,2]$ may be used as a source of new information on the origin of the 2.2-2.3 eV PL band. The microscopic nature of the defect responsible for the $2.2-2.3 \mathrm{eV}$ emission is uncertain, but most frequently it was attributed either to unintentional incorporation of copper on the zinc site, $\mathrm{Cu}_{\mathrm{Zn}}$, or to an excitation of a zinc vacancy, $\mathrm{V}_{\mathrm{Zn}_{\mathrm{n}}}$ [9]. We note, however, that the energy of this luminescence coincides with the position of the dominant deep level $\left(E_{2}\right)$ detected by DLTS at $0.51 \mathrm{eV}$ below the conduction band. This coincidence can be explained by assum- 
ing that the PL band originates from radiative transitions between the $E_{2}$ level and the valence band $\left(E_{\mathrm{g}}-0.51 \mathrm{eV}=2.2 \mathrm{eV}\right)$. Since DLTS results show that the $E_{2}$ defects are related to the presence of $\mathrm{Ga}$ atoms [1], it would then appear reasonable to attribute also $2.2-2.3 \mathrm{eV}$ PL band to Ga-containing complexes.

The PL results obtained on $\mathrm{Cl}$-doped $\mathrm{ZnSe}$ layers support the above assignment. In Cl-doped samples, the concentration of $\mathrm{Ga}$ impurities is relatively low, because they can originate only from the GaAs substrate. As a consequence, most of the zinc vacancies remain isolated. According to this, deep-level luminescence spectra are dominated by the $2.0 \mathrm{eV}$ band, which is due to isolated $\mathrm{V}_{\mathrm{Zn}_{\mathrm{n}}}$ [9]. Moreover, the intensity of the PL band is about 10 times weaker in Cl-doped samples than in Ga-doped specimens, reflecting the concentration ratio of $E_{2}$ defects in $\mathrm{ZnSe}: \mathrm{Cl}$ and $\mathrm{ZnSe}: \mathrm{Ga}$ layers (for layers with $n=3 \times 10^{17} \mathrm{~cm}^{-3}$ the concentration of $E_{2}$ in $\mathrm{ZnSe}: \mathrm{Ga}$ is $3 \times 10^{16} \mathrm{~cm}^{-3}$ [1], while in $\mathrm{ZnSe}: \mathrm{Cl} 5.3 \times 10^{15} \mathrm{~cm}^{-3}$ [2]).

Our photoluminescence data are in very good agreement with earlier studies on $\mathrm{Cl}$-doped $\mathrm{ZnSe}$ films [6]. In particular, they fully confirm the conclusion that, from the point of view of optical properties, $\mathrm{Cl}$ is superior to $\mathrm{Ga}$ as an $n$-type dopant.

\section{References}

[1] B. Hu, G. Karczewski, H. Luo, N. Samarth, J.K. Furdyna, Phys. Rev. B 47, 9641 (1993).

[2] G. Karczewski, B. Hu, H. Luo, J.K. Furdyna, J. Appl. Phys. 75, 7382 (1994).

[3] S. Venkatesan, R.F. Pierret, J. Qiu, M. Kobayashi, R.L. Gunshor, L.A. Kolodziejski, J. Appl. Phys. 66, 3656 (1989).

[4] T. Miyajima, K. Akimoto, Y. Mori, J. Appl. Phys. 67, 1389 (1990).

[5] J.M. DePuydt, H. Cheng, J.E. Potts, T.L.Smith, K. Mahapatra, J. Appl. Phys. 62, 4756 (1987).

[6] K. Ohkawa, T. Mitsuyu, O. Yamazaki, J. Appl. Phys. 62, 3216 (1987).

[7] J. Ren, K.A. Bowers, S. Hwang, J.W. Cook Jr., J.F. Schetzina, J. Cryst. Growth 111, 772 (1991).

[8] A. Kamata, T. Uemoto, K. Hirahara, T. Beppu, J. Appl. Phys. 65, 2561 (1989).

[9] J. Gutowski, N. Presser, G. Kudlek, Phys. Status Solidi A 120, 11 (1990) and references therein. 